\title{
Sex Differences in Antinociceptive Effects Induced by Gravity Stress in Rats
}

\author{
Mari Kimoto $^{1,2}$, Jorge Lopes Zeredo ${ }^{2,3}$, Zenro Nihei ${ }^{2}$, Hiromi Yamashita ${ }^{2}$, Kei Kaida ${ }^{3}$, Kazuo Toda ${ }^{2 *}$ \\ ${ }^{1}$ Physiological Laboratories, Japan Women's University, Tokyo, Japan \\ ${ }^{2}$ Integrative Sensory Physiology, Nagasaki University, Nagasaki, Japan \\ ${ }^{3}$ University of Brasilia, Campus Ceilandia, Brasilia, Brazil \\ Email: ${ }^{k}$ k-toda@nagasaki-u.ac.jp
}

Received March 9, 2013; revised April 10, 2013; accepted May 12, 2013

Copyright (C) 2013 Mari Kimoto et al. This is an open access article distributed under the Creative Commons Attribution License, which permits unrestricted use, distribution, and reproduction in any medium, provided the original work is properly cited.

\begin{abstract}
A number of studies have demonstrated that sex differentially affects responses to stress and pain. In this study, sex-related differences in pain responding were investigated in a gravity-induced analgesia model, where the effects of stressful high-gravity loading ( $1.5 \mathrm{G}$ or $2.0 \mathrm{G}$ for $10 \mathrm{~min})$ on nociceptive behavior in male and female rats were investigated. In each rat, eight sites (nose, both forepaws, upper and lower back, both hind paws and tail) were selected to apply noxious stimuli using a von Frey-type needle stimulator. The threshold values of the withdrawal responses were measured. In order to confirm the involvement of endogenous opioids in gravity-induced antinociceptive effects, naloxone- $\mathrm{HCl}$ (an opioid antagonist) was used. Effective analgesic effects could be induced by strong (2.0G) gravity loading, and clear sex differences were observed. Gravity-induced analgesic effects were more effective in males than in females, indicating that males are more sensitive to stress than females judging from nociceptive modulation. Naloxone-HCl produced a more pronounced suppression of nociceptive behavior in male rats, suggesting that gravity loading may activate endogenous opioids more readily in males than in females.
\end{abstract}

Keywords: Gravity; Stress; Sex; Nociception; Opioid; Rat

\section{Introduction}

Stress activates multiple neuronal and endocrine systems that modify pain sensation [1]. In particular, stress-induced analgesia (SIA) is well known to exist in various species of animals [2]. Various external stimuli, such as cold-water swimming [3-5], glucoprivation [6,7], footshock [8,9] and vaginal stimulation [10] can induce effective behavioral antinociception in rats. In human studies, it is reported that SIA is provoked by trauma [11], cold immersion [12] and psychological stress [2,13].

One of our previous studies [14] indicated that acute hypergravity stimulation can induce clear SIA in male rats; withdrawal threshold values in response to mechanical stimulation increased after $10 \mathrm{~min}$ of $2.0 \mathrm{G}$ (earth $\mathrm{G}$ $+1.0 \mathrm{G})$ gravity loading. Kumei et al. [15] reported that high gravity exposure upregulated Fos protein production in the hypothalamic arcuate nucleus of rats. Increased Fos expression in the central nervous system indicates a change in the excitability of synaptic transmission [16]. Because the arcuate nucleus is a key site for the hypo-

${ }^{*}$ Corresponding author. thalamus-pituitary-adrenal axis of the anti-stress system [17], hypergravity stimulation is thought to be a biological stressor that can alter nociceptive responses. Furthermore, gravity stimulation has been shown to increase plasma concentration of ACTH [18], supporting the view that gravity-loading is stressful and may alter the endocrine and central nervous systems.

Recently, sex has been increasingly recognized as one of the major variables to affect the sensitivity to stress [19-21]. For example, in response to stress, changes in feeding behavior, taste preference, and body weight are different between males and females [22,23]. Using coldwater swimming as a stressor, females tend to exhibit lower SIA than males [24]. However, there are no quantitative studies on sex differences in gravity-induced analgesia in experimental animals.

Considering above-mentioned background, it is interesting whether sex-related differences exist in the gravity-induced analgesia. Therefore, in the present study, we investigated the SIA induced by hypergravity loading and compared the patterns of SIA between male and female rats. 




(a)

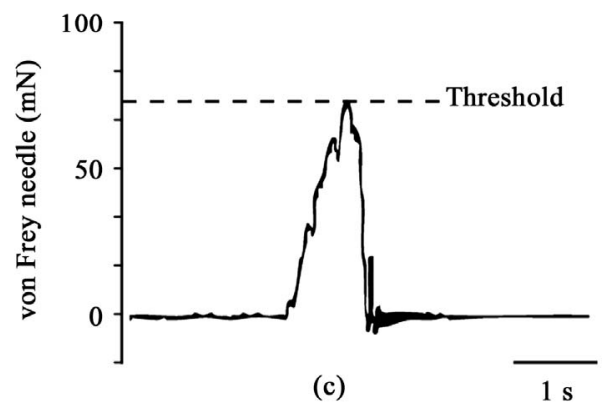

Figure 1. (a) Schematic drawing of the experimental setup. Noxious stimuli were applied with a von Frey-type needle attached to a strain gauge. The threshold values for a withdrawal reflex were measured and stored in a computer; (b) Stimulating site for evoking withdrawal responses (1: nose, 2: left forepaw, 3: right forepaw, 4: upper back, 5: lower back, 6: left hindpaw, 7: right hindpaw, 8: tail); (c) Example of strain gauge signal and measurement of threshold of withdrawal.

\section{Method}

\subsection{General}

The methods described here follow the ethical guidelines and received approval by the Animal Welfare Committee of Nagasaki University. Forty Wistar albino rats (male: 20, female: 20, Kyudo Animal Laboratories, Saga, Japan) weighing 130 - $146 \mathrm{~g}$ were randomly assigned to eight different groups (in each group, $\mathrm{n}=5$ rats): 1) $1.5 \mathrm{G}$ male group, 2) $1.5 \mathrm{G}$ female group, 3) $2 \mathrm{G}$ male group, 4) $2 \mathrm{G}$ female group, 5) Naloxone $+2 \mathrm{G}$ male group, 6) Naloxone $+2 \mathrm{G}$ female group, 7) saline $+2 \mathrm{G}$ male group, 8) saline $+2 \mathrm{G}$ female group. In the case of female rats, we used animals in the stage of proestrus. Vaginal impedance was checked by inserting bipolar electrodes (interpolar distance: $3 \mathrm{~mm}$ ) into vagina to determine proestrus, oestrus, metoestrus or dioestrus stage. According to Bartos
[25], vaginal impedance values over $3 \mathrm{~K} \Omega$ indicate that a female rat is in the proestrus stage.

It is generally accepted that repeated stress loading easily induces summation effects on the physiological responses [26,27]. To avoid these summative effects of gravity loading, each rat received gravity loading only once.

\subsection{Nociceptive Test and Gravity Loading}

In each rat, eight sites (nose, both forepaws, upper and lower back, both hindpaws and tail) were selected for the application of noxious stimuli by a von Frey-type needle stimulator [14] (Figure 1). The stimuli were applied randomly at each site in order to avoid sensitization of the skin. Measurements of mechanical force were performed with a force transducer (FORT-100, World Precision Instruments, Sarasota, FL, USA) attached to a von Freytype fine wooden needle. The signal of the mechanical force was amplified by a strain-gauge amplifier (TBM4M, World Precision Instruments, Sarasota, FL, USA) and stored in a personal computer through a CED interface (CED1401, Cambridge Electronic Design, Cambridge, UK). When animal escaped from the mechanical stimulus, a force curve showed a peak amplitude which represented the threshold of the withdrawal response. This value is regarded as a behavioral nociceptive threshold.

Before mounting in the centrifugal apparatus (AL-1, Kokusan Kogyo, Tokyo, Japan), the initial threshold values (baseline) required to evoke withdrawal responses were measured. Immediately after the baseline data was collected, the rats were loaded into the centrifugal apparatus for hypergravity stimulation. The rats were loaded individually into a mesh box $(160 \times 100 \times 65 \mathrm{~mm})$ facing the center of the centrifuge and stimulated an additional $0.5 \mathrm{G}$ (total $1.5 \mathrm{G}$ ) or $1.0 \mathrm{G}$ (total $2.0 \mathrm{G}$ ) for $10 \mathrm{~min}$. After cessation of gravity stimulation, the withdrawal thresholds were measured at fixed intervals for $90 \mathrm{~min}$.

\subsection{Naloxone Effects}

In order to confirm whether endogenous opioids were involved with the gravity-induced antinociceptive effects, naloxone-HCl (Sigma-Aldrich, St. Louis, MO, USA) was injected intraperitoneally in a dose of $0.1 \mathrm{mg} / \mathrm{kg}$. This dose of naloxone was reported to effectively antagonize stress-induced analgesia induced by peripheral nerve stimulation [28]. Naloxone was prepared at $0.1 \mathrm{mg} / \mathrm{ml}$ and injected at a dose of $1 \mathrm{ml} / \mathrm{kg}$. As a control, another group of rats received a volume of saline at $1 \mathrm{ml} / \mathrm{kg}$ injection. Five min after naloxone or saline injection, the baseline threshold in the naloxone and in the saline groups was measured. After that, 1.0G (total 2.0G) gravity loading was applied for $10 \mathrm{~min}$. 


\subsection{Data Analysis}

A time series of withdrawal thresholds was obtained before and after (base line and six time points from 0 to 90 $\min$ ) after $\mathrm{G}$ exposure. Time series data were analyzed using a split-plot ANOVA with a within-subjects factor of time ( 7 levels) and a between-subject factor of sex (male, female); in sites where statistically significant difference was found, one-way repeated measures ANOVA with Bonferroni post-hoc were used to determine differences in threshold between base-line and each time point, and differences between male and female at each time point. Naloxone time series was analyzed using a twoway repeated measures ANOVA with a within-subjects factor of time (7 levels) and a between-subject factors of sex (male, female) and drug (saline, naloxone). The ratio of antagonization, calculated as [AUC of the threshold values in Saline group from 0 to $90 \mathrm{~min}] /[$ AUC of threshold values in Naloxone group from 0 to $90 \mathrm{~min}$ ], was compared between male and female by the unpaired ttest. In all tests, we considered the significance level of $5 \%$. All data are expressed as means $\pm \mathrm{SE}$.

\section{Results}

\subsection{Threshold Values before Gravity Loading}

Withdrawal threshold values before gravity loading are shown in Figure 2, where baseline data from all groups were pooled together (male, $\mathrm{n}=20$ and female, $\mathrm{n}=20$ ). Control threshold value in the nose was significantly lower than that in the other seven sites both in males and females $(F(7,152)=14.711, p<0.0001$ in one-way factorial ANOVA followed by Bonferroni post-hoc test). No significant differences in baseline threshold values were observed between males and females at all stimulating sites. Therefore, sex differences in withdrawal threshold

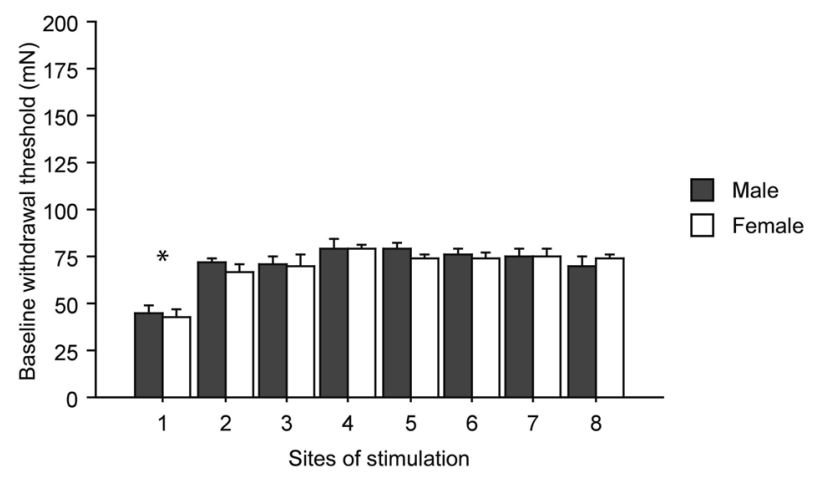

Figure 2. Baseline thresholds for withdrawal responses. On the horizontal axis are the sites of stimulation, from 1 to 8 , as shown in Figure 1. No statistically significant differences were found between male and female in any of the sites of stimulation before gravity-loading. Asterisk shows statistically significant difference between body sites in the one-way ANOVA with Bonferroni post-hoc. under physiological conditions were not detected.

\subsection{Weak (1.5G) Gravity Effects}

Time course of the threshold changes after $1.5 \mathrm{G}(+0.5 \mathrm{G})$ gravity loading is shown in Figure 3. Differences in the threshold of the withdrawal responses were detected between males and females over time. Split-plot ANOVA showed small but significant differences in threshold values in the left hindpaw $(\mathrm{F}(6,48)=2.770, \mathrm{p}=0.021)$, right hindpaw $(\mathrm{F}(2.692,21.535)=3.626, \mathrm{p}=0.033)$, and tail $(\mathrm{F}(6,48)=2.952, \mathrm{p}=0.015)$. In both hindpaws and tail, hyperalgesia was observed only in males (Figure 3).

\subsection{Strong (2.0G) Gravity Effects}

Time course of the threshold changes after $2 \mathrm{G}(+1 \mathrm{G})$ gravity loading is shown in Figure 4 . The split-plot ANOVA showed significant differences in threshold values between males and females in the nose $(\mathrm{F}(6,48)=$ 5.144, $\mathrm{p}<0.001)$ and in the left hindpaw $(\mathrm{F}(6,48)=$ $2.751, \mathrm{p}=0.022)$. Although the mean difference was big at several time points, the interaction between the effects time and sex did not reach statistical significance in the right forepaw $(\mathrm{F}(6,48)=1.169, \mathrm{p}=0.339)$. There were no significant sex differences in the threshold changes at the upper and lower back, hindpaws of both sides and tail. In general, gravity effects on the withdrawal responses showed their maximum at $30-50 \mathrm{~min}$ after cessation of stimulation and continued for $90 \mathrm{~min}$. In females, there was a significant increase in the withdrawal thresholds; however, the degree of gravity loading effects was smaller in females than in males. Changes in the threshold values at nose and both sides of forepaws were lower in females than in males. Particularly, sex difference was most pronounced in the threshold of withdrawal response induced by nose stimulation.

\subsection{Naloxone Effects}

There were no significant differences among baseline thresholds in $2 \mathrm{G}$ gravity groups, in the saline- $2 \mathrm{G}$ groups or in the naloxone- $2 \mathrm{G}$ groups. Therefore, naloxone alone did not alter the threshold value of the withdrawal responses before gravity stimulation. The two-way repeated measures ANOVA showed significant interaction between sex and drug over the time course of observation $(F(6,96)=2.357, p=0.036)$. The antinociceptive effects of the gravity loading were significantly antagonized by naloxone pretreatment both in males and females. In the nose, naloxone almost completely blocked antinociceptive effects of $2 \mathrm{G}$ gravity loading in males; whereas in females, naloxone antagonization was less effective as compared to that in males (Figure 5(a)). Direct comparison in reduction rate of naloxone on the 2 G-induced antinociception is shown in Figure 5(b). Antagonization 
ratio was significantly greater in males than that in fe-
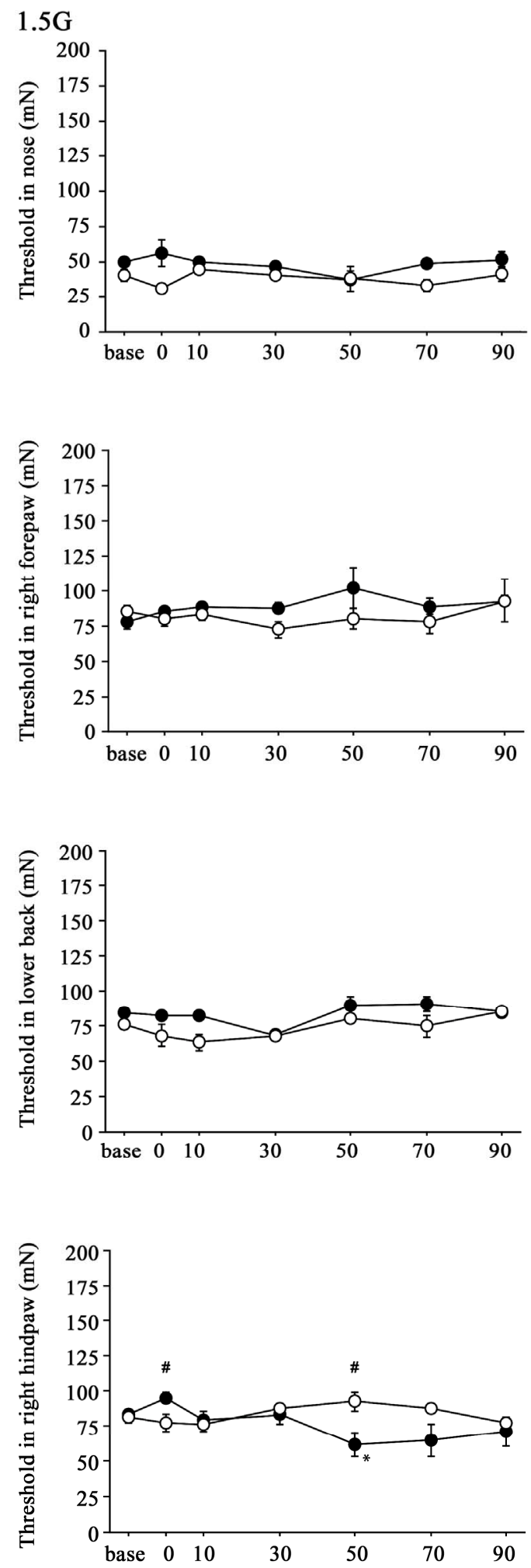

males $(\mathrm{p}<0.001$ in the unpaired t-test $)$.
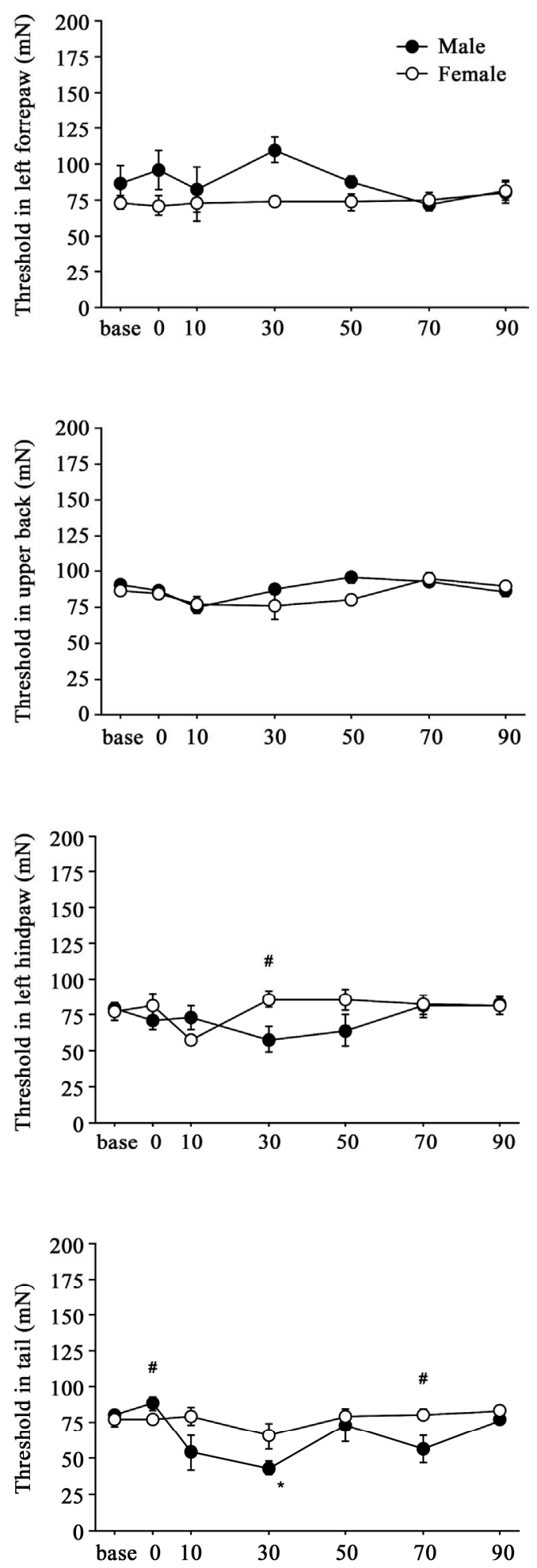

Figure 3. Time course of withdrawal threshold changes in eight stimulating sites before and after 1.5G gravity loading. Data are presented as mean $\pm \mathrm{SE}$. Asterisks show significant difference as compared with baseline threshold. "shows significant differences between males and females. Pairwise comparisons were done by one-way ANOVAs with Bonferroni post-hoc only for sites where the split-plot ANOVA showed statistically significant interaction between factors sex and time. 

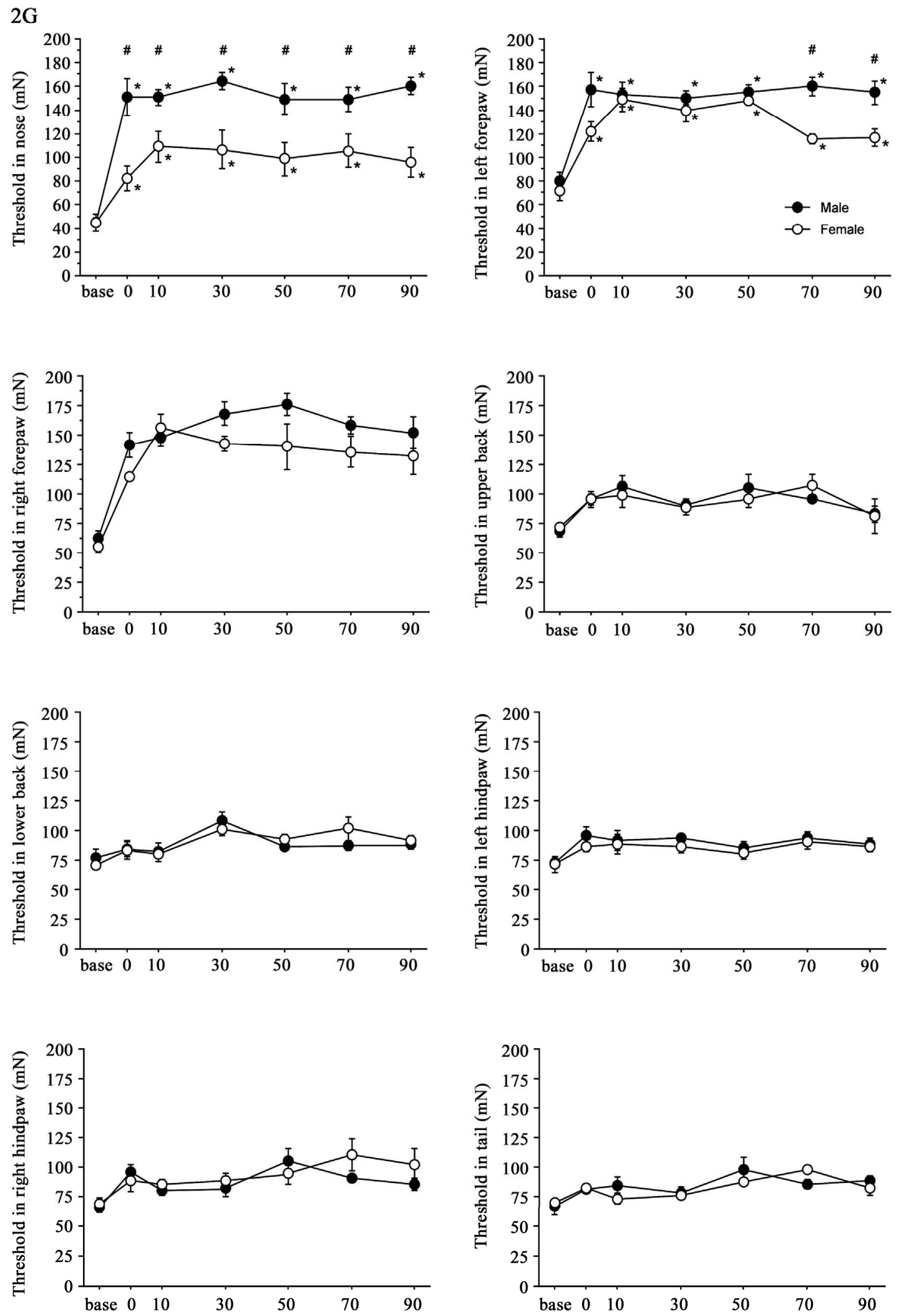

Figure 4. Time course of withdrawal threshold changes in eight stimulating sites before and after 2.0G gravity loading. Data are presented as mean \pm SE. Asterisks show significant difference as compared with baseline threshold. " shows significant difference between males and females. Pairwise comparisons were done by one-way ANOVAs with Bonferroni post-hoc only for sites where the split-plot ANOVA showed statistically significant interaction between factors sex and time. 


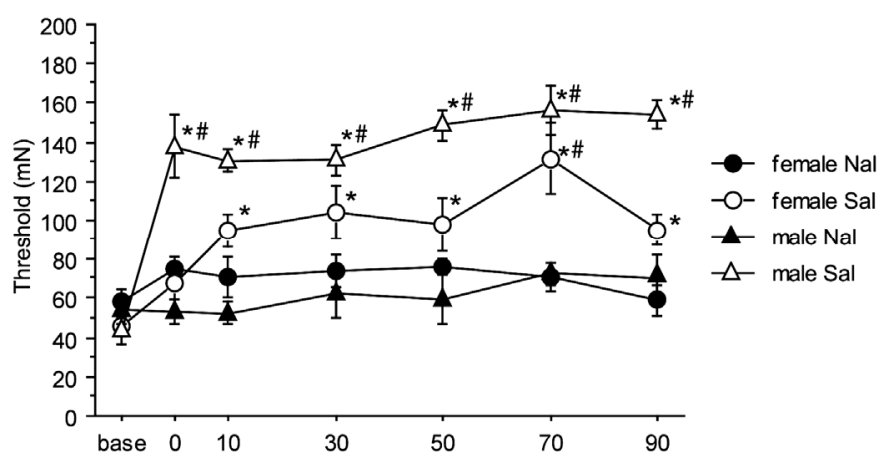

(a)

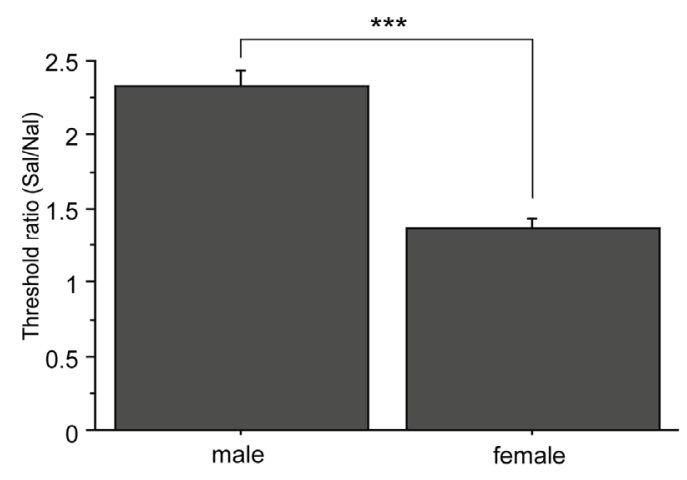

(b)

Figure 5. Naloxone effects on GIA. A: Time course of withdrawal threshold changes in nose after 2.0 G gravity loading. Saline or naloxone treatment was done 5 min before baseline threshold was recorded. The two-way repeated measures ANOVA showed statistically significant interaction between factors drug, sex, and time. * shows significant difference as compared with baseline threshold, and " shows significant difference as compared to the respective naloxone-group at each time point, in the one-way repeated measures ANOVA with Bonferroni post-hoc. B: Ratio of GIA antagonization by naloxone. The ratio was calculated as [AUC of the threshold values in Saline group from 0 to $90 \mathrm{~min}$ ]/[AUC of threshold values in Naloxone group from 0 to $90 \mathrm{~min}] .{ }^{* * *}$ shows significant difference $(\mathrm{P}<0.0001)$ in the unpaired t-test.

\section{Discussion}

The present study is the first report sex differences in SIA using gravity stress. Our results show that analgesic effects induced by strong (2.0G) gravity loading had significantly different magnitudes between male and female. In case of weak $(1.5 \mathrm{G})$ gravity loading, the analgesic effects were more diffuse and less pronounced, both in males and females. In $2 \mathrm{G}$ loading, gravity-induced analgesic effects were more effective in males than in females, indicating that males are either more sensitive to stress than females, or their SIA system is more readily activated after gravity stress. Naloxone-reversibility against gravity-induced analgesia was clearer in males than in females, suggesting that the non-opioid SIA system had a greater influence in females than in males.

In human studies, there is controversy about possible sex differences in pain perception, although it is generally accepted that women are more sensitive to pain than men [29-31]. Nevertheless, females report more pain than males at the first exposure to pain, but then experience less pain than males as a painful stimulus is repeated. Moreover, sex differences in pain sensation are not uniform across the various types of nociceptive stimuli, such as heat, cold, and mechanical stimulation [32]. Therefore, it remains unclear whether sex differences exist in pain sensitivity in humans $[33,34]$. Like in human studies, sex differences in the sensitivity to noxious stimulation were observed in rats $[35,36]$. In rat studies, different methods of experimental noxious stimulation, such as mechanical, thermal, or chemical, may have produced inconsistencies between studies in nociceptive sensitivity in males and females. In the present study, we used quantitative mechanical stimulation to evoke nociceptive responses. Without gravity loading, sex differences in the withdrawal re- sponses were not observed in any of the stimulated sites.

\subsection{Weak (1.5G) Gravity Stress}

In the present study, $1.5 \mathrm{G}$ gravity loading did not cause analgesic effects. On the contrary, decreased withdrawal thresholds were observed in males, but not in females. It is reported that brief exposure to vibration-stress $(4 \mathrm{~Hz}$ for $5 \mathrm{~min}$ ) causes hyperalgesia in rats, as evidenced by a decrease in tail flick latency [37]. This kind vibration may be regarded as a mild, non-noxious type of stressor. The same study reported that, among male rats, about $50 \%$ show hyperalgesia, and the other $50 \%$ show a brief hypoalgesia. It is conceivable that the $1.5 \mathrm{G}$ stress used in the present study may have been a mild stressor, like vibration, thus causing ambiguous results on the sensitivity to pain.

\subsection{Strong (2.0G) Gravity-Stress}

2.0G gravity stress had clear analgesic effects on the withdrawal responses. The magnitudes of the analgesic effects were stronger in males than in females. Sex differences in withdrawal responses were seen in the nose and in the forepaws on both sides.

There are several lines of evidence for sex differences in pain perception induced by noxious stimuli in humans and experimental animals. Generally, females are thought to be more sensitive than males in response to pain [29, $38,39]$. In the present study, sex differences were more clearly in body sites where gravity-stimulation induced greater analgesic effect. For instance, the nose, which is innervated by the second branch of the trigeminal nerve, was the most effective site for gravity induced analgesia (GIA). Therefore, it is presumed that stress-modified trigeminal pain sensation may strongly depend on sex. A 
similar interaction has been reported in relation to taste sensation during food intake [40].

\subsection{Naloxone Effects}

Analgesic effects induced by $2.0 \mathrm{G}$ gravity loading were well antagonized by naloxone with clear sex differences. The degree of antagonization was greater in males than in females. It has been suggested that multiple neuronal mechanisms are involved in endogenous pain modulation [41]. SIA in particular comprises an opioid and a nonopioid system. It has also been reported that both opioid and non-opioid forms of SIA are elicited in rodents according to the type of stressor and to the duration of exposure to that stressor $[4,42]$. Although endogenous opioids appear to be key mediators in the inhibition of nociceptive response to stressful stimuli, recent studies now support the existence of SIA mechanisms that do not depend on the opioid system. It is apparent that a certain type of SIA is not reversed by naloxone [9,42]. Thus, evidence has accumulated that other neurohumoral networks including GABAergic, glutamatergic and monoaminergic systems play an important role in mediating non-opioid SIA [2]. In addition, mechanisms of SIA involve various neuroendocrine systems, most importantly the hypothalamus-pituitary-adrenal system [43]. In females, ovarian hormones additionally act as a modulator for SIA, which may be included in the non-opioid SIA system [44]. Nevertheless, in this study, the gravity-induced effects were completely antagonized by naloxone pretreatment in both males and females, indicating that the opioid system was mostly responsible for GIA in rats, with a greater effect in males than in females. In conclusion, it is suggested that gravity loading may activate the endogenous opioids system more readily in males than in females.

\section{Acknowledgements}

This study was partly supported by JSPS grants to KT (2007-2009, 2010-2012).

\section{REFERENCES}

[1] N. I. Yarushkina, "The Role of Hypothalamo-HypophysealAdrenocortical System Hormones in Controlling Pain Sensitivity," Neuroscience and Behavioral Physiology, Vol. 38, No. 8, 2008, pp. 759-766. doi:/10.1007/s11055-008-9044-z

[2] R. K. Butler and D. P. Finn, "Stress-Induced Analgesia," Progress in Neurobiology, Vol. 88, No. 3, 2009, pp. 184-202. doi:/10.1016/j.pneurobio.2009.04.003

[3] L. Aloe, P. Tirassa and E. Alleva, "Cold Water Swimming Stress Alters Ngf and Low-Affinity Ngf Receptor Distribution in Developing Rat Brain," Brain Research Bullutin, Vol. 33, No. 2, 1994, pp. 173-178.

\section{doi:/10.1016/0361-9230(94)90247-X}

[4] R. J. Bodnar, D. D. Kelly, M. Brutus and M. Glusman, "Stress-Induced Analgesia: Neural and Hormonal Determinants," Neuroscience Biobehavior Review, Vol. 4, No. 1, 1980, pp. 87-100. doi:/10.1016/0149-7634(80)90028-7

[5] M. N. Girardot and F. A. Holloway, "Intermittent Cold Water Stress-Analgesia in Rats: Cross-Tolerance to Morphine," Pharmacology Biochemistry and Behavior, Vol. 20, No. 4, 1984, pp. 631-633. doi:/10.1016/0091-3057(84)90315-0

[6] R. J. Bodnar, D. D. Kelly, M. Brutus, A. Mansour and M. Glusman, "2-Deoxy-D-Glucose-Induced Decrements in Operant and Reflex Pain Thresholds," Pharmacology Biochemistry and Behavior, Vol. 9, No. 4, 1978, pp. 543549. doi:/10.1016/0091-3057(78)90056-4

[7] R. McGivern, C. Berka, G. G. Berntson, J. M. Walker and C. A. Sandman, "Effect of Naloxone on Analgesia Induced by Food Deprivation," Life Science, Vol. 25, No. 10, 1979, pp. 885-888. doi:/10.1016/0024-3205(79)90547-2

[8] H. Akil, J. Madden, R. L. Patrick and J. D. Barchas, "Stress-Induced Increase in Endogenous Opiate Peptides: Concurrent Analgesia and Its Partial Reversal by Naloxone," In: H. W. Kosterlitz, Ed., Opiates and Endogenous Opioid Peptides, North-Holland Publishing Co., Amsterdam, 1976, pp. 63-70.

[9] J. W. Lewis, J. T. Cannon and J. C. Liebeskind, "Opioid and Nonopioid Mechanisms of Stress Analgesia," Science, Vol. 208, No. 4444, 1980, pp. 623-625. doi:/10.1126/science.7367889

[10] W. R. Crowley, J. F. Rodriguez-Sierra and B. R. Komisaruk, "Analgesia Induced by Vaginal Stimulation in Rats Is Apparently Independent of a Morphine-Sensitive Process," Psychopharmacology (Berl), Vol. 54, No. 3, 1977, pp. 223-225. doi:/10.1007/BF00426567

[11] P. Nishith, M. G. Griffin and T. L. Poth, "Stress-Induced Analgesia: Prediction of Posttraumatic Stress Symptoms in Battered Versus Nonbattered Women," Biological Psychiatry, Vol. 51, No. 11, 2002, pp. 867-874. doi:/10.1016/S0006-3223(01)01346-4

[12] L. Knudsen and P. D. Drummond, "Cold-Induced Limb Pain Decreases Sensitivity to Pressure-Pain Sensations in the Ipsilateral Forehead," European Journal of Pain, Vol. 13, No. 10, 2009, pp. 1023-1029. doi:/10.1016/j.ejpain.2008.12.005

[13] P. M. Aslaksen and M. A. Flaten, "The Roles of Physiological and Subjective Stress in the Effectiveness of a Placebo on Experimentally Induced Pain," Psychosomatic Medicine, Vol. 70, No. 7, 2008, pp. 811-818. doi:/10.1097/PSY.0b013e31818105ed

[14] Y. Kawauchi, J. L. Zeredo, Y. Takeuchi, M. Kimoto, Y. Kumei, K. Makita and K. Toda, "Nociceptive Threshold Can Be Changed by Gravity Stimulation in Rats," Stress and Health, Vol. 20, No. 1, 2004, pp. 45-50. doi:/10.1002/smi.990

[15] Y. Kumei, R. Shimokawa, M. Kimoto, Y. Kawauchi, H. Shimokawa, K. Makita, K. Ohya and K. Toda, "Gravity Stress Elevates the Nociceptive Threshold Level with Immunohistochemical Changes in the Rat Brain," Acta Astronaut, Vol. 49, No. 3-10, 2001, pp. 381-390. 
[16] K. J. Kovacs, "Measurement of Immediate-Early Gene Activation-C-Fos and Beyond," Journal of Neuroendocrinology, Vol. 20, No. 6, 2008, pp. 665-672. doi:/10.1111/j.1365-2826.2008.01734.x

[17] A. Sainsbury and L. Zhang, "Role of the Arcuate Nucleus of the Hypothalamus in Regulation of Body Weight during Energy Deficit," Molecular Cell Endocrinology, Vol. 316, No. 2, 2010, pp. 109-119. doi:/10.1016/j.mce.2009.09.025

[18] L. Macho, R. Kvetnansky, M. Fickova, I. A. Popova and A. Grigoriev, "Effects of Exposure to Space Flight on Endocrine Regulations in Experimental Animals," Endocrine Regulations, Vol. 35, No. 2, 2001, pp. 101-114.

[19] R. M. Craft, J. S. Mogil and A. M. Aloisi, "Sex Differences in Pain and Analgesia: The Role of Gonadal Hormones," European Journal of Pain, Vol. 8, No. 5, 2004, pp. 397-411. doi:/10.1016/j.ejpain.2004.01.003

[20] W. F. Sternberg and J. C. Liebeskind, "The Analgesic Response to Stress: Genetic and Gender Considerations," European Journal of Anaesthesiology-Supplement, Vol. 10, No. 1995, pp. 14-17.

[21] G. J. Ter Horst, R. Wichmann, M. Gerrits, C. Westenbroek and Y. Lin, "Sex Differences in Stress Responses: Focus on Ovarian Hormones," Physiological Behaviour, Vol. 97, No. 2, 2009, pp. 239-249. doi:/10.1016/j.physbeh.2009.02.036

[22] M. M. Faraday, K. H. Blakeman and N. E. Grunberg, "Strain and Sex Alter Effects of Stress and Nicotine on Feeding, Body Weight, and Hpa Axis Hormones," Pharmacology Biochemistry and Behavior, Vol. 80, No. 4, 2005, pp. 577-589. doi:/10.1016/j.pbb.2005.01.015

[23] D. E. Pankevich and T. L. Bale, "Stress and Sex Influences on Food-Seeking Behaviors," Obesity (Silver Spring), Vol. 16, No. 7, 2008, pp. 1539-1544. doi:/10.1038/oby.2008.221

[24] M. T. Romero and R. J. Bodnar, "Gender Differences in Two Forms of Cold-Water Swim Analgesia," Physiological Behaviour, Vol. 37, No. 6, 1986, pp. 893-897. doi:/10.1016/S0031-9384(86)80009-9

[25] L. Bartos, "Vaginal Impedance Measurement Used for Mating in the Rat," Lab Animal, Vol. 11, No. 1, 1977, pp. 53-55. doi:/10.1258/002367777780959148

[26] C. Chotiwat, E. W. Kelso and R. B. Harris, "The Effects of Repeated Restraint Stress on Energy Balance and Behavior of Mice with Selective Deletion of Crf Receptors," Stress, Vol. 13, No. 3, 2010, pp. 203-213. doi:/10.3109/10253890903207527

[27] H. Imbe, K. Okamoto, T. Donishi, E. Senba and A. Kimura, "Involvement of Descending Facilitation from the Rostral Ventromedial Medulla in the Enhancement of Formalin-Evoked Nocifensive Behavior Following Repeated Forced Swim Stress," Brain Research, Vol. 1329, No. 2010, pp. 103-112. doi:/10.1016/j.brainres.2010.03.006

[28] K. Toda, H. Suda, M. Ichioka and A. Iriki, "Local Electrical Stimulation: Effective Needling Points for Suppressing Jaw Opening Reflex in Rat," Pain, Vol. 9, No. 2, 1980, pp. 199-207. doi:/10.1016/0304-3959(80)90007-X

[29] K. J. Berkley, "Sex Differences in Pain," Behavior Brain
Science, Vol. 20, No. 3, 1997, pp. 371-380. doi:/10.1017/S0140525X97221485

[30] R. B. Fillingim, "Sex, Gender, and Pain: Women and Men Really Are Different," Current Review Pain, Vol. 4, No. 1, 2000, pp. 24-30. doi:/10.1007/s11916-000-0006-6

[31] R. B. Fillingim and W. Maixner, "Gender Differences in the Responses to Noxious Stimuli," Pain Forum, Vol. 4, No. 4, 1995, pp. 209-221.

[32] J. L. Riley, M. E. Robinson, E. A. Wise, C. D. Myers and R. B. Fillingim, "Sex Differences in the Perception of Noxious Experimental Stimuli: A Meta-Analysis," Pain, Vol. 74, No. 2-3, 1998, pp. 181-187. doi:/10.1016/S0304-3959(97)00199-1

[33] R. B. Fillingim, C. D. King, M. C. Ribeiro-Dasilva, B. Rahim-Williams and J. L. Riley, "Sex, Gender, and Pain: A Review of Recent Clinical and Experimental Findings," Journal of Pain, Vol. 10, No. 5, 2009, pp. 447-485. doi:/10.1016/j.jpain.2008.12.001

[34] J. A. Hashmi and K. D. Davis, "Women Experience Greater Heat Pain Adaptation and Habituation Than Men," Pain, Vol. 145, No. 3, 2009, pp. 350-357. doi:/10.1016/j.pain.2009.07.002

[35] S. G. Khasar, F. J. Miao, R. W. Gear, P. G. Green and J. D. Levine, "Vagal Modulation of Bradykinin-Induced Mechanical Hyperalgesia in the Female Rat," Journal of Pain, Vol. 4, No. 5, 2003, pp. 278-283. doi:/10.1016/S1526-5900(03)00631-X

[36] C. J. Vierck, A. J. Acosta-Rua, H. L. Rossi and J. K. Neubert, "Sex Differences in Thermal Pain Sensitivity and Sympathetic Reactivity for Two Strains of Rat," Journal of Pain, Vol. 9, No. 8, 2008, pp. 739-749. doi:/10.1016/j.jpain.2008.03.008

[37] A. J. Devall and T. A. Lovick, "Differential Activation of the Periaqueductal Gray by Mild Anxiogenic Stress at Different Stages of the Estrous Cycle in Female Rats," Neuropsychopharmacology, Vol. 35, No. 5, 2010, pp. 11741185. doi:/10.1038/npp.2009.222

[38] M. Frot, J. S. Feine and M. C. Bushnell, "Sex Differences in Pain Perception and Anxiety. A Psychophysical Study with Topical Capsaicin," Pain, Vol. 108, No. 3, 2004, pp. 230-236. doi:/10.1016/i.pain.2003.11.017

[39] J. M. Tall and T. Crisp, "Effects of Gender and Gonadal Hormones on Nociceptive Responses to Intraplantar Carrageenan in the Rat," Neuroscience Letters, Vol. 354, No. 3, 2004, pp. 239-241. doi:/10.1016/j.neulet.2003.09.081

[40] M. Kimoto, J. L. Zeredo and K. Toda, "Irritant-Drinking Behaviour Can Be Modified by Gravity-Stress Loaded in Developing but Not in Adult Rats," Stress and Health, Vol. 27, No. 1, 2011, pp. 34-41. doi:/10.1002/smi.1317

[41] M. Lafrance, G. Roussy, K. Belleville, H. Maeno, N. Beaudet, K. Wada and P. Sarret, "Involvement of Nts2 Receptors in Stress-Induced Analgesia," Neuroscience, Vol. 166, No. 2, 2010, pp. 639-652. doi:/10.1016/j.neuroscience.2009.12.042

[42] L. R. Watkins, D. A. Cobelli, P. Faris, M. D. Aceto and D. J. Mayer, "Opiate Vs Non-Opiate Footshock-Induced Analgesia (Fsia): The Body Region Shocked Is a Critical Factor," Brain Research, Vol. 242, No. 2, 1982, pp. 299-308. 
doi:/10.1016/0006-8993(82)90313-4

[43] J. McBeth, Y. H. Chiu, A. J. Silman, D. Ray, R. Morriss, C. Dickens, A. Gupta and G. J. Macfarlane, "Hypothalamic-Pituitary-Adrenal Stress Axis Function and the Relationship with Chronic Widespread Pain and Its Antece- dents," Arthritis Research Therapy, Vol. 7, No. 5, 2005, pp. R992-R1000. doi:/10.1186/ar1772

[44] V. T. Martin, "Ovarian Hormones and Pain Response: A Review of Clinical and Basic Science Studies," Gender Medicine, Vol. 6, No. 2009, pp. 168-192. 